Supporting Information

For

\title{
Preparation of a Millimeter-Sized Supergiant Liposome That Allows for Efficient, Eukaryotic Cell-Free Translation in the Interior by Spontaneous Emulsion Transfer
}

\author{
Hajime Takahashi and Atsushi Ogawa* \\ Proteo-Science Center, Ehime University, 3 Bunkyo-cho, Matsuyama, Ehime 790-8577, Japan
}

\section{CONTENTS:}

Sequence of Synthetic Nucleic Acids (p. S2)

Supplementary Figures. (p. S3) 


\section{Sequences of Synthetic Nucleic Acids.}

(written from 5' (left) to 3' (right); shaded: SP6 or T7 promoter; X = 2'-OMe-G; ds: double-stranded; ss: single-stranded)

Forward primer for amplifying dsDNA encoding YPet

CATACGATTTAGGTGACACTATAGAAC

Reverse primer for amplifying dsDNA encoding YPet

TTAGCGGCTTTATTGATTGC

Forward primer in the 1st PCR for amplifying dsDNA encoding Broccoli

GAAATTAATACGACTCACTATAGGGAGACCACAACGGTTTCCCGCGGAGACGGTCGGGT CCAGATATTCG

Reverse primer in the 1st PCR for amplifying dsDNA encoding Broccoli

TGGCGGAGAGAGAGGGATTCGCCCTCGCACCACTCGAGCATCCGCCGCGGAGCCCACAC TCTACTC

Template ssDNA in the 1st PCR for amplifying dsDNA encoding Broccoli

CGGTTTCCCGCGGAGACGGTCGGGTCCAGATATTCGTATCTGTCGAGTAGAGTGTGGGCT CCGCGGCGGATGCT

Forward primer in the 2nd (final) PCR for amplifying dsDNA encoding Broccoli

GAAATTAATACGACTCACTATAG

Reverse primer in the 2nd (final) PCR for amplifying dsDNA encoding Broccoli

TXGCGGAGAGAGAGGGATTC 


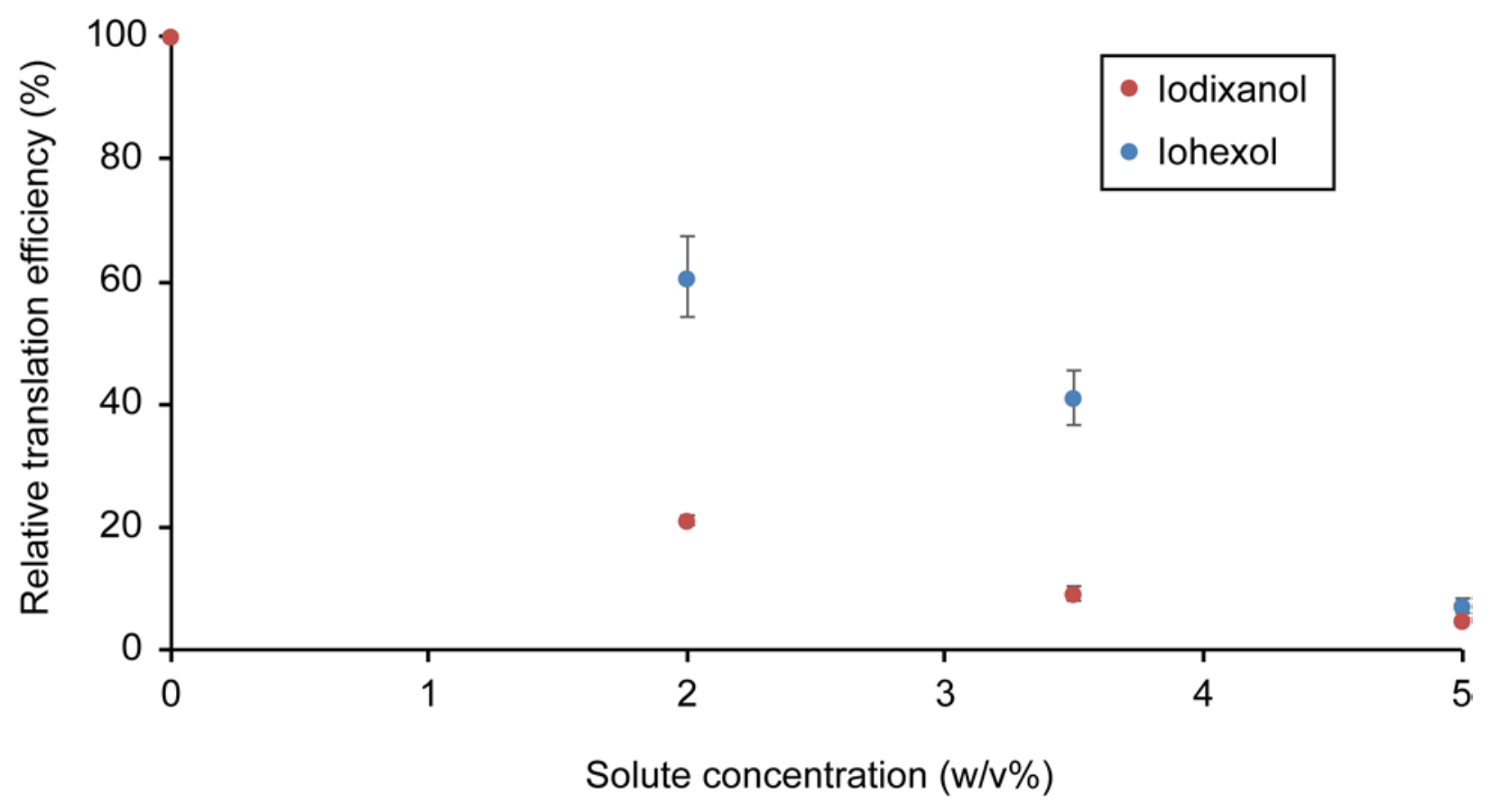

Figure S1. Relative translation efficiency of YPet-encoding mRNA in the presence of various concentrations of nonsugar high-density solutes (iodixanol and iohexol) in the WGE translation system. The translation efficiency was evaluated with the fluorescence intensity of translated YPet. 


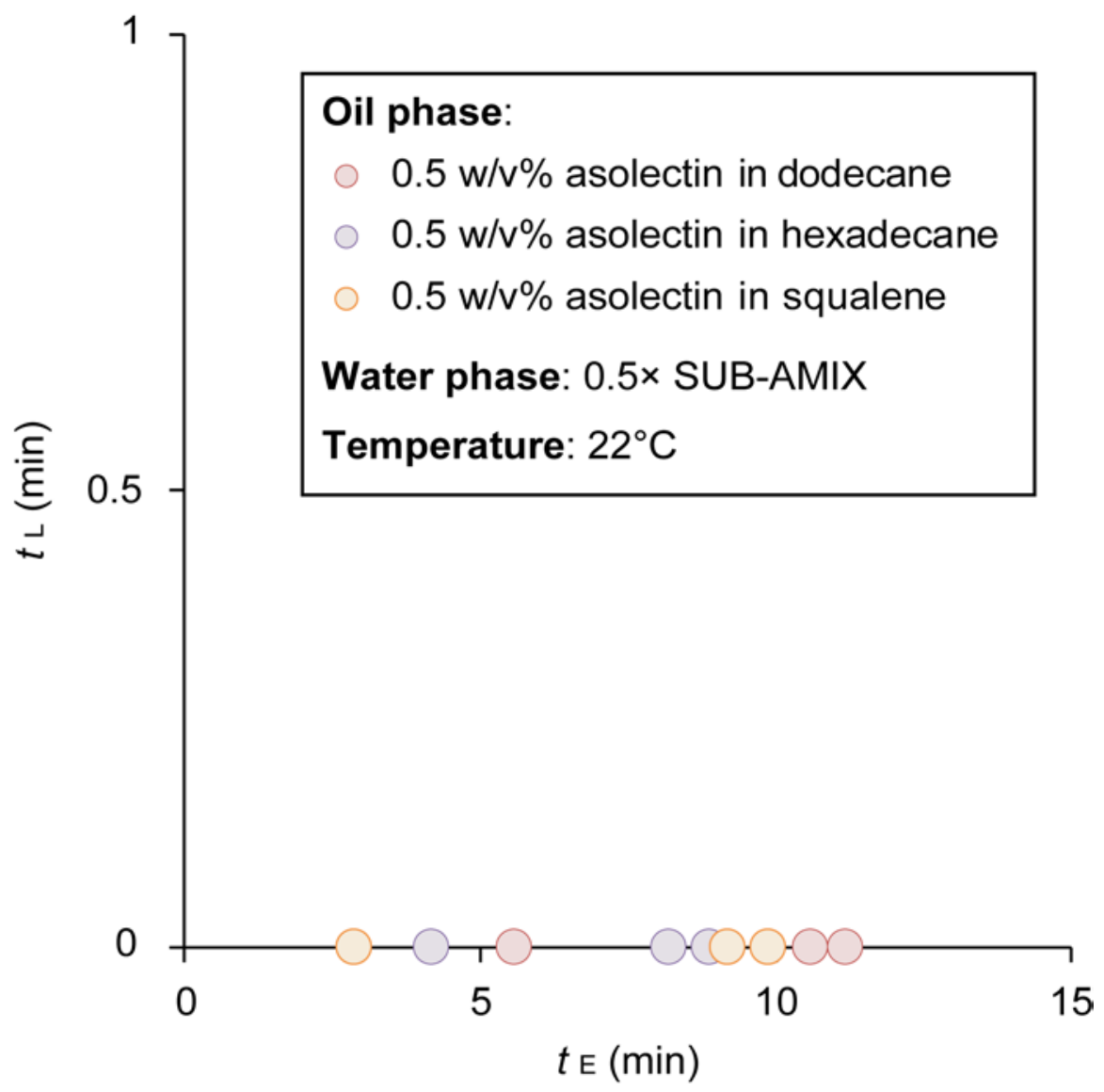

Figure S2. Trials of SGUV formation with dodecane, hexadecane, or squalene including $0.5 \mathrm{w} / \mathrm{v} \%$ asolectin in the oil phase and 0.5× SUB-AMIX in the water phase. The $x$-axis $\left(t_{\mathrm{E}}\right)$ and the $y$-axis $\left(t_{\mathrm{L}}\right)$ in each graph represent how long each droplet existed as an emulsion (or incomplete liposome) and a complete liposome, respectively. No successful SGUV formation was observed. 
(A)
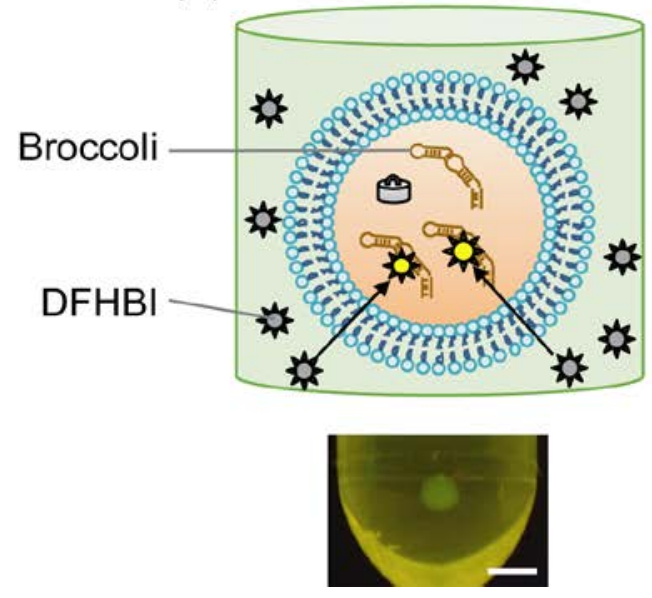

(B)
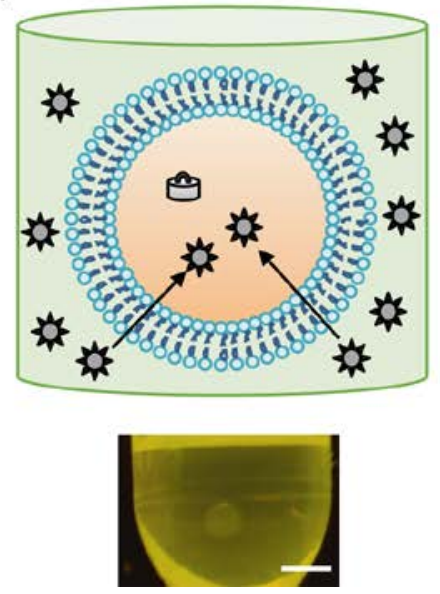

(C)
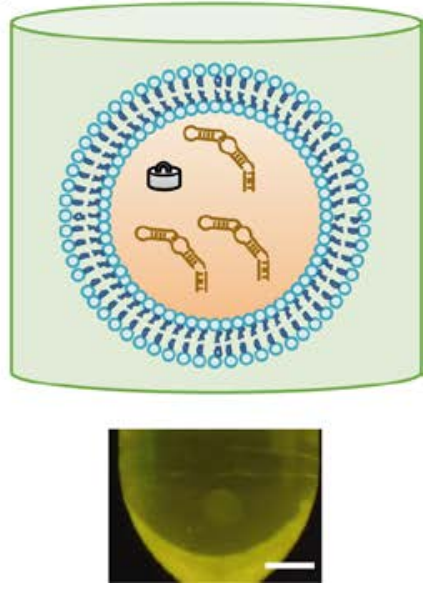

Figure S3. Schematic illustration (above) and fluorescence images (below) of a Broccoli-encapsulating SGUV with $100 \mu \mathrm{M}$ DFHBI in the exterior (A), a Broccoli-free SGUV with $100 \mu \mathrm{M}$ DFHBI in the exterior (B), and a Broccoli-encapsulating SGUV without DFHBI in the exterior (C). The images were acquired approximately $1 \mathrm{~h}$ after the addition of a droplet to the oil phase. The latter two showed no fluorescence, meaning that the green fluorescence in the foremost image surely reflects the binding between Broccoli and permeated DFHBI in the interior. Scale bar: $2.5 \mathrm{~mm}$. 


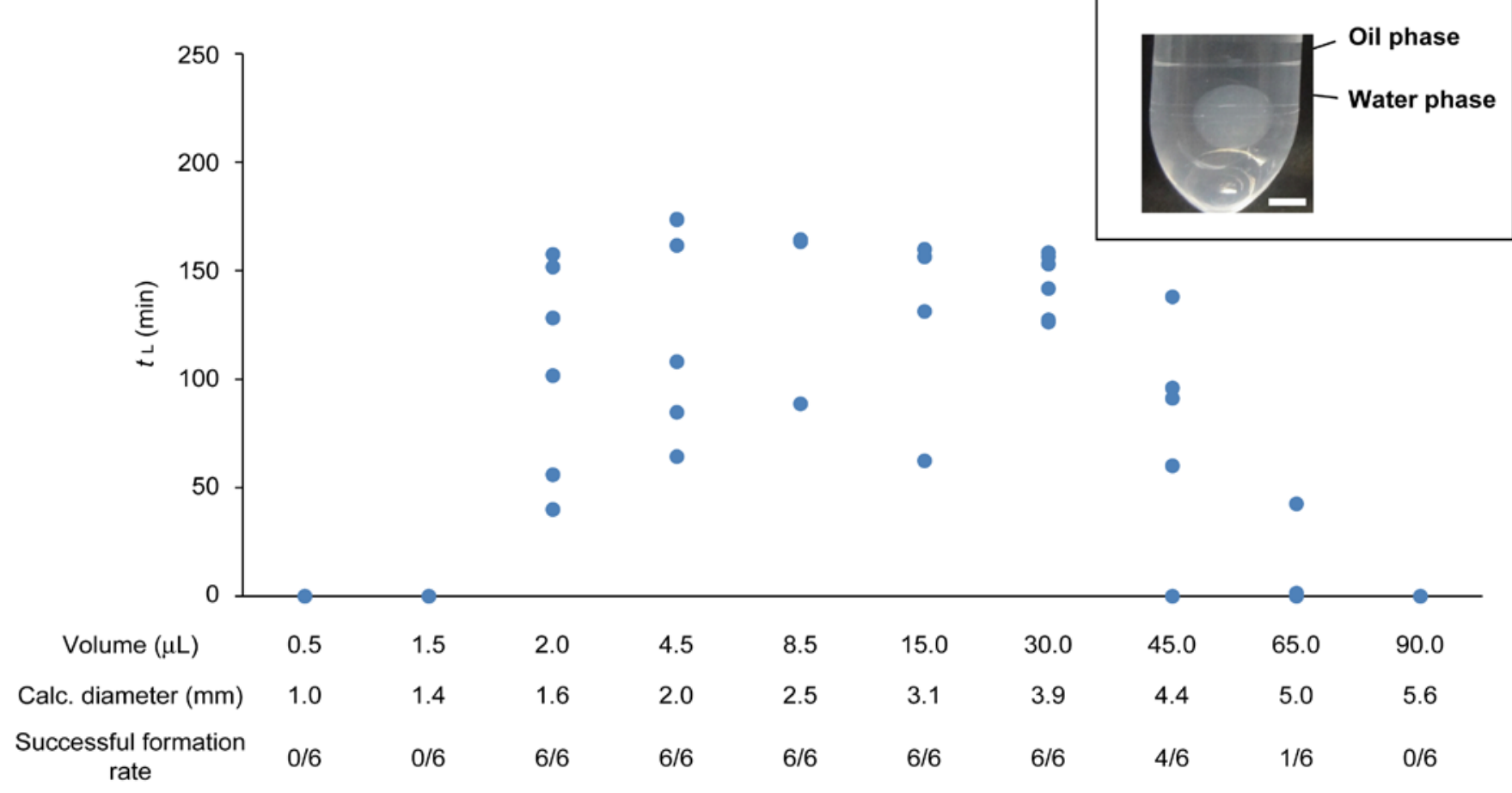

Figure S4. Preparation of various volume sizes of SGUVs under the optimized conditions. The $y$-axis represents how long each SGUV existed. The number of trials is 6 in each size and the successful formation rate is described below the graph. As a successful example, an image of $45-\mu \mathrm{L}$ SGUV is shown in the inset (scale bar: $2.5 \mathrm{~mm}$ ). Emulsion droplets smaller than $1.5 \mu \mathrm{L}$ were not able to transfer through the oil/water interface due to being too light to overcome the interfacial tension. In contrast, emulsion droplets larger than $65 \mu \mathrm{L}$ burst during the phase transfer probably because their surface area is comparable to or wider than the area of the oil/water interface (approximately $78.5 \mathrm{~mm}^{2}$ ). 